\title{
Performances obtenues sur SU5 : une ligne VUV à haute résolution et à polarisation variable
}

\author{
L. Nahon ${ }^{1,2}$ \\ ${ }^{1}$ LURE, bâtiment 209D, Université Paris Sud, BP. 34, 91898 Orsay cedex, France \\ ${ }^{2}$ CEAIDRECAM/SPAM, CE de Saclay, bâtiment 522, 91191 Gif-sur-Yvette cedex, France
}

\begin{abstract}
Résumé : La ligne de lumière VUV SU5 répond à un double cas scientifique : la spectroscopie à haute résolution sur des especces diluées et le dichroïsme linéaire/circulaire sur des systèmes anisotropes. Un onduleur croisé plan/hélicoîdal électromagnétique a été conçu et construit, produisant des polarisations variables avec un fort flux. Nous avons montres la grande polyvalence des états de polarisation de la lumière produite, avec des taux de polarisation élevés. Par ailleurs, les harmoniques de l'onduleur peuvent être supprimées grâce à un filtre à gaz, agissant comme un filtre passe-bas en énergie, avec des taux de réjection supérieurs à $10^{5}$. La ligne est centrée autour d'un monochromateur à incidence nomale du type "Eagle off-plane" qui a atteint des pouvoirs résolvants bruts sans précédent $(117000$ a $21.6 \mathrm{eV}, 133000$ à $15.8 \mathrm{eV})$ avec des flux typiques dans une bande passante de 1/50000 de l'ordre de $10^{10} \mathrm{a} 10^{9}$ entre 6 et $30 \mathrm{eV}$.
\end{abstract}

\section{INTRODUCTION}

La ligne SU5 de Super-ACO [1] est initialement dédiée à l'étude, dans le domaine VUV, de la spectroscopie à très haute résolution et des processus induits par impacts photoniques sur des espèces diluées. Un second cas scientifique, s'intéresse aux systèmes anisotropes, via des expériences de dichroïsme linéaire et circulaire, nécessitant l'usage de "polarisations exotiques", i.e. linéaire tournante et circulaire. SU5 est la seule ligne VUV visant à la fois la haute résolution et le dichroïsme. Certaines lignes, telle la ligne Chemical Dynamics Facility (CDF) de l'Advanced Light Source (ALS) basée sur un onduleur plan [2,3], ont obtenues de la haute résolution avec un fort flux grâce à des monochromateurs (pouvoir résolvant de 70000), tandis que d'autres ont utilisé des spectromètres à réseaux fixes pour faire des expériences d'absorption à ultra-haute résolution (pouvoir résolvant de 250000) [4]. Par ailleurs, les seules lignes VUV avec "polarisations exotiques" se sont jusqu'à présent limitées au seul usage du Rayonnement Synchrotron (RS) hors du plan produit dans un aimant de courbure [5] avec un compromis entre le flux et le taux de polarisation.

Afin de réaliser le double programme scientifique de SU5, la ligne devait se plier aux nécessités techniques suivantes : (i) la possibilité de générer des "polarisations exotiques"; (ii) une haute pureté spectrale; (iii) un pouvoir résolvant ultime de l'ordre de $10^{5}$ entre 5 et $30 \mathrm{eV}$ avec un fort flux. Les différents éléments de la ligne permettant de remplir ces contraintes sont un onduleur croisé électromagnétique, un filltre à gaz et un monochromateur à incidence normal (NIM) de $6,65 \mathrm{~m}$, tels qu'ils apparaissent sur la Figure 1, et tels qu'ils sont décrit ci-après.

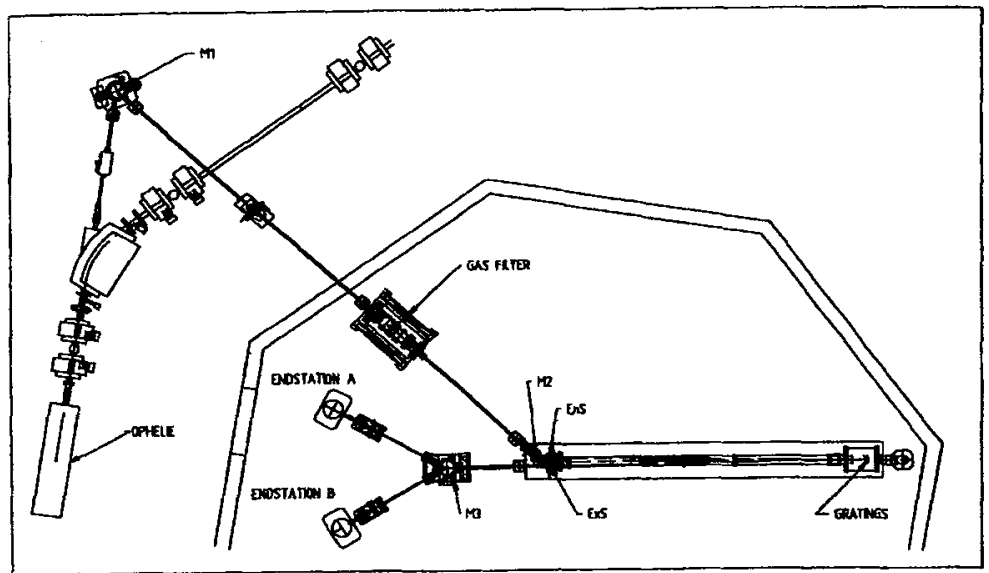

Figure 1. Schéma de la ligne SUS située au centre de Super-ACO. EnS / ExS correspondent à fente d'entrée/sortie. 


\section{L'ONDULEUR OPHELIE}

Afin d'obtenir à la fois du flux et de forts taux de polarisation, nous avons conçu un onduleur spécifique, appelé OPHELIE (Onduleur Plan/Hélicoïdal du Lure à Induction Electromagnétique), comme source de RS pour SU5 [6]. D'abord l'optimisation de sa structure magnétique pour le domaine VUV, nous a conduit à choisir un design avec 10 longues périodes de $25 \mathrm{~cm}$ et un faible champ magnétique $(\max .0,12 \mathrm{~T})$. Ceci limite à moins de $2 \mathrm{~W}$ la puissance déposée sur les première optiques. Dans une deuxième étape, nous avons optimisé la génération de n'importe quel type d'état de polarisation du RS, afin d'obtenir sur l'échantillon de la polarisation linéaire tournante et circulaire.

Nous avons ainsi construit pour la première fois à grande échelle et sur un centre serveur de RS, un onduleur croisé du type Onuki [7]. De plus, considérant la structure magnétique choisie, et par soucis de polyvalence, nous avons opté pour une technologie électro-magnétique. OPHELIE est constitué de deux onduleurs plans croisés identiques produisant un champ vertical $\left(\mathrm{BV}_{\mathrm{V}}\right)$ et un champ horizontal $\left(\mathrm{B}_{\mathrm{H}}\right)$, indépendamment accordable en faisant varier leur courant d'alimentation, et dont le déphasage longitudinal peut être ajusté grâce au seul mouvement mécanique de translation. On peut montrer qu'il est possible de générer n'importe quel état de polarisation sur le fondamental, repéré par ses paramètre de Stokes, en jouant sur les 3 paramètres $\left(\mathrm{B}_{\mathrm{V}}\right),\left(\mathrm{B}_{\mathrm{H}}\right)$ et $(\phi)$ [6]. En inversant la polarité de $\left(\mathrm{B}_{\mathrm{V}}\right)$ avec une alimentation bipolaire, on inverse les signes des paramètres de Stokes $S_{2}$ et $S_{3}$, permettant de réaliser directement des expériences de dichroïsme linéaire et circulaire.

Après une mise en oeuvre sous faisceau validée en mode continu (DC : un basculement de polarité en une minute) [8], les propriétés du RS émis ont été caractérisées à l'aide d'un monochromateur de $0,3 \mathrm{~m}$ suivi d'un polarimètre VUV à 4 réflexions. L'analyse de la polarisation, effectuée à $7 \mathrm{eV}$, confirme la grande polyvalence d'OPHELIE, puisque pour la première fois dans le domaine VUV, il a été possible de produire et de mesurer une très large variété d'ellipses de polarisation, incluant bien sûr les cas linéaire et circulaire. Quantitativement, en mode polarisation linéaire, le paramètre $S_{1}$ est supérieur à $95 \%$, tandis qu'en mode polarisation circulaire, il a été possible d'obtenir un taux de polarisation circulaire normalisé proche de $100 \%$, avec une valeur absolue (préliminaire) de $S_{3}$ supérieure à $80 \%$ [9].

\section{LE FILTRE GAZ}

Un des problèmes fréquemment rencontré par les utilisateurs du RS est la présence d'ordres élevés de diffraction par les réseaux qui ne sont pas séparés du premier ordre au vu de la condition de Bragg. Cette contribution d'ordre élevés/harmoniques élevées peut, dans certains cas, cacher ou surpasser le signal d'intérêt. Nous avons donc décidé de développer un filtre passe-bas en énergie.

Alors que dans le l'UV, il existe des matériaux solides transparents tels que le $\mathrm{MgF} 2$ ou le LiF avec des coupures vers 10 et $12 \mathrm{eV}$, il n'y a pas de matériaux transparents dans le domaine VUV. Nous avons alors utilisé une cellule d'absorption à gaz, dont le principe est d'absorber tout photons dont l'énergie est au dessus du seuil d'ionisation du gaz considéré, par exemple 15,7 eV avec de l'argon et 21,6 eV avec du néon, selon une idée initialement développée sur la ligne CDF de l'ALS [10]. Un simple calcul, basé sur la loi de Beer-Lambert, montre qu'avec une pression de 1 mbar et une longueur d'absorption de $10 \mathrm{~cm}$, on peut obtenir des facteurs d'atténuations supérieurs à $10^{4}$.

Le défi technologique réside ici dans la nécessité de conserver un vide très poussé (typiquement de l'ordre de $10^{-11}$ mbar) dans la chambre à vide de l'anneau située $12 \mathrm{~m}$ en amont du filtre à gaz. Afin d'assurer un tel pompage différentiel, nous avons séparer les étages "haute pression", "moyenne pression" et "basse pression" par des capillaires (2,5 mm de diamètre, $10 \mathrm{~cm}$ de long) limitant la conductance. La forme précise de la cellule à gaz (zone "haute pression") a été optimisée par des simulations numériques en régime intermédiaire (entre les régimes laminaire et moléculaire) fournissant notamment la distribution de pression dans un capillaire [11].

Les résultats engrangés sont très satisfaisants, puisque comme cela avait été simulé, nous avons pu injecter jusqu'à 1 mbar d'Ar et 0,5 mbar de Ne sans augmentation mesurable de la pression partielle du gaz rare correspondant dans la chambre à vide de l'anneau. L'efficacité du filtre à gaz a été mesurée, en terme d'atténuation, par des mesures d'absorption et de rendement d'ions. Le résultat le plus marquant et très satisfaisant est que nous avons pu obtenir une atténuation des harmoniques d'un facteur supérieur à 200000 vers $16 \mathrm{eV}$ avec 0,6 mbar d'Ar, sans aucune atténuation du fondamental [11]. On dispose ainsi d'un rayonnement pur, se prêtant à tout type de spectroscopie.

\section{LES ELEMENTS OPTIQUES ET LE MONOCHROMATEUR}

Le design optique est centré autour d'un monochromateur à incidence normale du type "Eagle off-plane" de $6,65 \mathrm{~m}$ de longueur focale, éclairé par un système de pré-focalisation astigmatique, et équipé de deux réseaux sphériques, fabriqués par holographie, G1 $(2400 \mathrm{tr} / \mathrm{mm})$ et $\mathrm{G} 2(4300 \mathrm{tr} / \mathrm{mm})$ couvrant respectivement les domaines 5-20 eV et $12-40 \mathrm{eV}$ [12]. Le RS blanc est dispersé par les réseaux via deux mouvements couplés de rotation et de translation afin de rester sur le cercle de Rowland. Avec les très 
hautes résolutions visées, ces deux mouvements couplés conduisent à des contraintes très sévères en termes de précision et de stabilité mécanique. Il est à noter qu'un soin particulier a été porté concemant les points critiques susceptibles de limiter la résolution spectrale ultime tels que les vibrations statiques et dynamiques, la conception du système de contrôle électronique des moteurs/codeurs, ainsi que l'optimisation de la résolution mécanique de la "barre sinus" (environ $10 \mathrm{~nm}$ ) qui entraîne la rotation des réseaux.

Afin de mesurer la résolution ultime, nous avons enregistré des spectres d'autoionisation de gaz rares, dans la région des hauts états de Rydberg à longue durée de vie. Sur la Figure 2, est représenté un tel spectre dans le cas du $\mathrm{Ne}$, enregistré avec des fentes de $18 \mu \mathrm{m}$, et montrant les deux séries $\mathrm{s}^{\prime}$ et $\mathrm{d}^{\prime}$ convergeant vers le deuxième seuil d'ionisation. Comme ia durée de vie de ces états est longue, on peut mesurer directement une largeur instrumentale de $220 \mu \mathrm{eV}$, conduisant à un pouvoir résolvant de 97000 [13]. En encart est présenté le spectre de la raie 18s,' enregistré avec des fentes de $8 \mu \mathrm{m}$, accompagné d'une courbe d'ajustement gaussien. La largeur à mi-hauteur correspondante est de $184 \mu \mathrm{eV}$, conduisant à un pouvoir résolvant de 117000 à $21,6 \mathrm{eV}$, au-dessus de la plus haute valeur publiée à ce jour, 70000 , obtenue sur la ligne CDF de l'ALS [3].

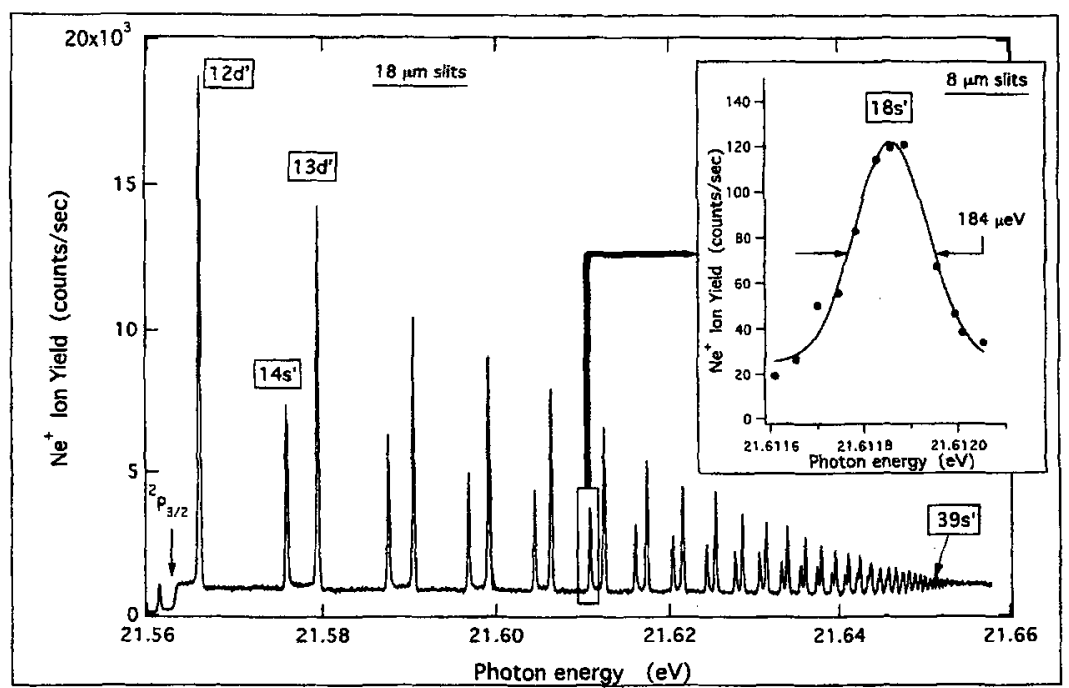

Figure 2. Spectre d'autoionisation du Ne, enregistre entre les deux seuils $2 \mathrm{p}^{-1}$, avec des fentes de $18 \mu \mathrm{m}$, montrant une résolution de $220 \mu \mathrm{eV}$ (pouvoir résolvant de 97000). En encart : ( () spectre de la raie $18 \mathrm{~s}^{\prime}$ enregistré avec des fentes de $8 \mu \mathrm{m}$; $(\longrightarrow$ Ajustement gaussien montrant une largeur à mi-hauteur de $184 \mu \mathrm{eV}$ (pouvoir résolvant de 117000).

Plusieurs autres mesures de la résolution ultime ont été effectuées sur l'argon et le xénon, pour chacun des deux réseaux et à différentes énergies de photons [13]. Les résultats bruts et déconvolués de la durée de vie sont présentés sur le Tableau 1. A notre connaissance, ce jeux de données montre les plus forts pouvoirs résolvants jamais atteints par un monochromateur dans le domaine VUV. L'observation de la variation de la résolution spectrale en fonction de l'ouverture des fentes montre que jusqu'à 15-18 $\mu \mathrm{m}$ (resp. 10-12 $\mu \mathrm{m}$ ) d'ouverture de fente, pour G2 (resp. G1), la résolution est uniquement limitée par les fentes selon l'approximation géométrique, dans laquelle la résolution spectrale est simplement donnée par la dispersion spectrale dans le plan de la fente de sortie, i.e. $0,335 \AA / \mathrm{mm}$ (resp. $0,6 \AA / \mathrm{mm}$ ). En dessous, on est essentiellement limité par les erreurs de pentes, $0,47 \mu \mathrm{rad}$ pour $\mathrm{Gl}$ et $0,8 \mu \mathrm{rad}$ pour G2. Dans tous les cas, les vibrations semblent négligeables puisqu'il n'est pas nécessaire d'introduire leur contribution pour rendre compte des résolutions atteintes.

Tableau 1. Résolutions ultimes en énergie ( $\Delta \varepsilon$ ), brutes et déconvoluées de la durée de vie, avec les pouvoirs résolvants (PR) correspondants (PR) pour les deux réseaux, mesurées par rendement d'ion sur des raies autoionisantes.

\begin{tabular}{|c|c|c|c|c|c|c|}
\hline & Raie & $\varepsilon(\mathrm{eV})$ & $\Delta \varepsilon$ brute $(\mu \mathrm{eV})$ & PR brut & Déconv $\Delta \varepsilon(\mu \mathrm{eV})^{2}$ & Déconv. PR \\
\hline \multirow{2}{*}{$(2400 \mathrm{tr} / \mathrm{mm})$} & $\mathrm{Xe} 16 \mathrm{~s}^{\prime}$ & 13.34 & 144 & 92000 & 100 & 133000 \\
\cline { 2 - 7 } & $\mathrm{Ar} 13 \mathrm{~s}^{\prime}$ & 15.82 & 141 & 112000 & 111 & 142000 \\
\hline \multirow{3}{*}{$\mathrm{G} 2$} & $\mathrm{Xe} 16 \mathrm{~s}^{\prime}$ & 13.34 & 104 & 128000 & 71 & 188000 \\
\cline { 2 - 7 }$(4300 \mathrm{tr} / \mathrm{mm})$ & $\mathrm{Ar} 13 \mathrm{~s}^{\prime}$ & 15.82 & 119 & 133000 & 76 & 208000 \\
\cline { 2 - 7 } & $\mathrm{Ne} 18 \mathrm{~s}^{\prime}$ & 21.61 & 184 & 117000 & - & - \\
\hline
\end{tabular}

${ }^{1}$ Les valeurs déconvoluées de la durée de vie sont obtenues par ajustement d'un profil de Voigt dans lequel la contribution lorentzienne possède une largeur correspondant à la durée de vie publiée du niveau. 
Le flux de photons moyen, dans une bande passante donnée, est avec la résolution ultime, un paramètre important. Clairement, au vu du spectre de la Figue 2, montrant un très bon rapport signal/bruit, on peut inférer que le flux délivré par SU5 est assez élevé. De façon plus quantitative, nous avons mesuré le flux de photons incident sur l'échantillon à l'aide du courant de photoemission produit par une grille d'or située an aval du demier miroir M3. Cette mesure a été effectuée à différentes énergies de photons pour un pouvoir résolvant fixe de 50000 , nécessitant un réglage correspondant de l'ouverture des fentes. Le flux typique mesuré dans une bande passante de 1/50000, tel qu'il apparait sur la Figure 3, se situe dans la gamme $10^{10}$ à $10^{9}$ entre 6 et $30 \mathrm{eV} \mathrm{[13],} \mathrm{en} \mathrm{accord} \mathrm{raisonnable} \mathrm{avec} \mathrm{les} \mathrm{performances} \mathrm{attendues} \mathrm{[12].}$

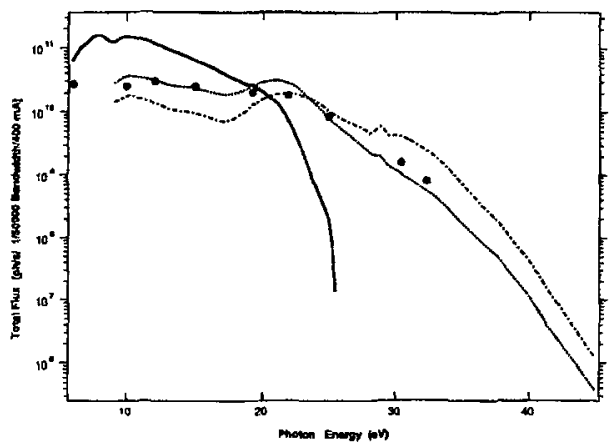

Figure 3. Flux de photon sur l'échantillon dans une bande spectrale de 1/50000:(*) Mesures; (-), (....), (--) Calculs selon différentes combinaisons des optiques (M1 et réseaux).

\section{CONCLUSIONS}

La nouvelle ligne SU5 à haute résolution et à polarisation variable, a respecté le cahier des charges qui lui était assigné : l'onduleur croisé OPHELIE produit des photons avec une grande polyvalence de l'état de polarisation et de forts taux de polarisation; un filtre à gaz par absorption assure un rayonnement débarrassé de ses harmoniques et des pouvoirs résolvants sans précédents, supérieur à $10^{5}$ sur la majeur partie du domaine spectral considéré, ont été mesurés. Après une période de 4 ans, dédiée à la conception, la construction et la mise en oeuvre de la ligne, autant d'étapes qui ont mobilisé une charge de travail interne au LURE de 40 homme.ans, SU5 accueille ses premiers utilisateurs depuis mars 2000 . L'activité future va se tourner vers la mise en cuvre de l'onduleur sous faisceau en mode altematif (AC : $0,1-0,5 \mathrm{~Hz}$ ) ainsi que sur le développement d'un nouveau polarimètre VUV dédié à la ligne, qui permettra à tout moment, in situ et sous vide, d'analyser l'état de polarisation de RS juste en amont de l'échantillon.

\section{Références}

[1] Voir aussi : http://www.lure.u-psud.fr/Experiences/SACO/SU5/intro_SU5.htm

[2] Koike M., Heimann P. A., Kung A. H., Namioka T., DiGennaro G., Gee B. et Yu N., Nucl. Instrum. Meth A 347 (1994) 282-286.

[3] Heimann P.A., Koike M., Hsu C.W., Evans M., Ng C.Y., Blanck D., Yang X.M., Flaim C., Suits A.G. et Lee Y.T., Proc. SPIE 2856 (1996) 90-99.

[4] Ito K., Namioka K., Morioka Y., Sasaki T., Noda H., Goto K., Katayama T. et Koike M., Appl. Opt. 25 (1986) 837-847.

[5] Voir par exemple Schäfers F., Peatman W., Eyers A., Heckenkamp C., Schönhense G. et Heinzmann U., Rev. Sci. Instr. 57 (1986) 1032-1041.

[6] Nahon L., Corlier M., Peaupardin P., Marteau F., Marcouillé O., Brunelle P., Alcaraz C. et Thiry P., Nucl. Instrum. Meth. A 396 (1997) 237-250.

[7] Onuki H., Nucl. Instrum. Meth. A 246 (1986) 94-98.

[8] Nahon L., Peaupardin P., Marteau F., Marcouillé O., Brunelle P., Thissen R., Alcaraz C. et Corlier M., Nucl. Instrum. Meth. A 447 (2000) 569-586.

[9] Alcaraz C., Thissen R., Compin M., Jolly A., Drecher M. et Nahon L., Proc. SPIE 3773 (1999) 250 261.

[1.0] Suits A., Heimann P., Yang X., Evans M., Hsu C., Lu T., Lee Y.T. et Kung A. H., Rev. Sci. Instr. 66 (1995) 4841-4844.

[11] Mercier B., Compin M., Prevost C., Bellec G., Thissen R., Dutuit O. et Nahon L., J. Vac. Sci. Technol. A 18 (sept 2000) (sous presse).

[12] Nahon L., Lagarde B., Polack F., Alcaraz C., Dutuit O., Vervloet M. et Ito K., Nucl. Instrum. Meth. A 404 (1998) 418-429.

[13] Nahon L., Alcaraz C., Marlats J.-L., Lagarde B., Polack F., Thissen R., Lepère D. et Ito K., soumis à Rev. Sci. Instr. 